\section{Condição de saúde bucal de idosos institucionalizados em Goiânia- GO, 2003}

\section{Oral health status of institutionalized elderly in Goiânia- GO, Brazil, 2003}

\section{Sandra Cristina Guimarães Bahia Reis ${ }^{{ }^{*}}$}

Maria Abadia Salge Prata Higino'

Hugo Montalvão Dias de Melo'

Maria do Carmo Matias Freire ${ }^{2}$

'Departamento de Saúde Bucal, Secretaria Municipal de Saúde de Goiânia ${ }^{2}$ Departamento de Ciências Estomatológicas, Faculdade de Odontologia, Universidade Federal de Goiás

\section{Resumo}

Objetivo: conhecer a condição de saúde bucal de idosos institucionalizados na cidade de Goiânia-GO. Método: estudo transversal utilizando a metodologia do Projeto SB-2000, Brasil do Ministério da Saúde. A amostra foi constituída de todos os indivíduos com idade igual ou maior que 60 anos ( $\mathrm{n}=289)$ residentes nas instituições públicas e filantrópicas de longa permanência da cidade de Goiânia-GO (n=10) em 2003. As condições estudadas foram cárie, uso e necessidade de prótese, doença periodontal e alterações de tecido mole. Resultados: A faixa etária variou de 60 a 103 anos. As prevalências de cárie e edentulismo foram 100\% e 69,20\%, respectivamente. O CPOD médio foi 30,17, havendo predomínio do componente extraído. Quase a metade $(49,48 \%)$ usava e $80,28 \%$ necessitavam de alguma prótese. O tipo de prótese mais comum foi a total: $45,33 \%$ dos examinados usavam este tipo de prótese na arcada superior e $24,57 \%$ na inferior; enquanto $59,17 \%$ e $51,21 \%$ necessitavam na arcada superior e inferior, respectivamente. De acordo com o Índice Periodontal Comunitário (CPI), apenas $3(3,37 \%)$ dos idosos apresentaram todos os sextantes sadios. A pior condição mais freqüente foi o cálculo, observado em $55,06 \%$ dos indivíduos e $29,02 \%$ dos sextantes. Quanto ao Índice de Perda de Inserção Periodontal (PIP), houve predomínio das perdas de 0 a $3 \mathrm{~mm}$ como pior condição em $37,08 \%$ dos indivíduos e $24,04 \%$ dos sextantes. Alterações de tecido mole foram encontradas em 13,49\% dos indivíduos. Conclusão: A situação de saúde bucal dos idosos institucionalizados em Goiânia é precária, especialmente devido à alta prevalência de cárie e edentulismo.

Palavras-chave: Idoso. Saúde bucal. Epidemiologia

*Correspondência: Av. 5a. Radial, Qd. 216 A , Lt. 5, Setor Pedro Ludovico, 74823-030 Goiânia- 
Abstract

Objective: To assess the oral health status of the institutionalized elderly in the city of Goiânia in the state of Goiás, Brazil. Method: A cross-sectional study was carried out based on the SB-2000 Project undertaken by the Brazilian Ministry of Health. The sample was formed by people aged 60 years and above ( $\mathrm{n}=289$ ), living in Goiânia in public and philanthropic institutions for the elderly during 2003 ( $n=10)$. Conditions investigated included dental caries, use of and need for prostheses, periodontal status, and soft tissue lesions. Results: The age of the elderly involved in the study ranged from 60 to 103 years. Prevalence of caries and edentulousness was $100 \%$ and $69.20 \%$ respectively. The mean DMFT (decayed, missing and filled teeth) totaled $30.17 \%$ and, of these, tooth extraction was the main component. Nearly half (49.48\%) of the sample was using prostheses and $80.28 \%$ were in need of some form of it. The most common type of prosthesis was complete denture: $45.33 \%$ of the individuals were using them in the upper and $24.57 \%$ in the lower maxilla; whilst $59.17 \%$ and $51.21 \%$ needed them in the upper and lower maxilla respectively. According to the Community Periodontal Index (CPI), only 3 of the elderly (3.37\%) had all sextants sound. The most serious condition was calculus, found in $55.06 \%$ of individuals and in $29.02 \%$ of the sextants. Regarding the Attachment Loss Index (ALI), the most common condition was loss from 0 to $3 \mathrm{~mm}$ in $37.08 \%$ of the individuals and in $24.04 \%$ of the sextants. Soft tissue lesions were found in $13.49 \%$ of the elderly. Conclusion: The oral health status of institutionalized elderly in Goiânia is poor, mainly due to the high prevalence of caries and edentulism.

Key Words: Elderly. Oral health. Epidemiology.

\section{Introdução}

Com o envelhecimento populacional observado no mundo e no Brasil, tem crescido a demanda por serviços e instituições para o atendimento e acompanhamento integral ao idoso.

O estatuto do idoso prioriza o atendimento em sua própria família em detrimento do atendimento asilar, exceto nos casos em que este e sua família carecerem de condições mínimas de sobrevivência ${ }^{1}$.

A internação do idoso em uma instituição de longa permanência pode se apresentar como única saída para a família, frente à não disponibilidade do suporte familiar, financeiro e psicológico que o mesmo necessita $^{2}$. Nestas instituições, o indivíduo vive na forma de internato, por tempo determinado ou não, mediante pagamento ou não ${ }^{3}$.

Nas duas últimas décadas, diversos estudos sobre idosos foram realizados em municípios brasileiros ${ }^{4,5}$, revelando altos índices de cárie e edentulismo. A maioria destes referem-se ao Estado de São Paulo a partir dos anos 1990, e poucos foram realizados em indivíduos institucionalizados. Mello ${ }^{6}$ investigou o cuidado odontológico provido em instituições de pequeno porte de Porto Alegre e verificou que o índice de placa foi alto entre os indivíduos examinados, embora os supervisores e cuidadores considerassem que o cuidado odontológico fosse realizado com regularidade.

O primeiro inquérito nacional realizado no Brasil e que avaliou as condições de saúde bucal de idosos não institucionalizados foi o Projeto SB-2000, Brasil ${ }^{7}$, coordenado pelo Ministério da Saúde em 2003. A amostra incluiu indivíduos de 18-36 meses a 65-74 anos de 250 municípios, incluindo Goiânia, e foi calculada para ser representativa das cinco macro-regiões do Brasil.

O objetivo do presente estudo é conhecer a condição de saúde bucal de idosos com idade igual ou maior que 60 anos, residentes nas instituições públicas e filantrópicas de longa permanência da cidade de Goiânia, para subsidiar a formulação de um programa municipal de saúde voltado para a população resi- 
dente naquelas instituições. Este trabalho foi proposto também em cumprimento a Política Nacional do Idoso ${ }^{8}$, que determina a realização de estudos epidemiológicos, com vistas à prevenção, tratamento e reabilitação.

\section{Métodos}

O presente estudo constitui um subprojeto do SB-2000, Brasil 7 . Foi realizado nas instituições públicas e filantrópicas de longa permanência da cidade de Goiânia-GO no segundo trimestre de 2003. Um total de dez instituições foi identificado na listagem disponibilizada pela Divisão de Doenças Crônico Degenerativas da Secretaria Municipal de Saúde de Goiânia. A população de estudo incluiu todos os indivíduos com 60 anos de idade ou mais, de ambos os sexos, independentes, parcialmente dependentes e dependentes, segundo a classificação da Federação Dentária Internacional ${ }^{9}$ e que estivessem presentes no momento do exame, em condições de serem examinados.

A metodologia utilizada foi proposta pelo Projeto SB-2000, Brasil ${ }^{7}$, o qual foi aprovado pela Comissão Nacional de Ética em Pesquisa (CONEP) através do Parecer No 581/2000. A realização do presente subprojeto foi aprovada pela Secretaria Municipal de Saúde e instituições participantes. Somente os idosos que autorizaram o exame, através de um termo de consentimento livre e esclarecido, foram examinados. No caso de idosos analfabetos ou incapacitados para assinar, o termo foi assinado pelos responsáveis das instituições. Os indivíduos que apresentaram alterações de tecido mole foram encaminhados para diagnóstico e tratamento no Centro Goiano de Doenças da Boca (CGDB) da Faculdade de Odontologia da Universidade Federal de Goiás.

A coleta de dados foi feita através de exames clínicos em agosto de 2003. Foi realizada por cinco equipes, cada uma composta por um Cirurgião-Dentista (examinador) e um Técnico de Higiene Dental (anotador) da Secretaria Municipal de Saúde de Goiânia, os quais participaram da coleta de dados do Projeto SB-2000, Brasil ${ }^{7}$ naquele município, após treinamento e calibração. As condições estudadas foram: a) cárie dentária e necessidade de tratamento, através do Índice CPOD (número de dentes cariados, perdidos e restaurados); b) condição periodontal, através do Índice Periodontal Comunitário (CPI) e do Índice de Perda de Inserção Periodontal (PIP); c) uso e necessidade de próteses total, fixa e removível; e d) alteração de tecidos moles (ausência ou presença). Os critérios de diagnóstico foram aqueles do Projeto SB2000, Brasil .

Os exames foram realizados na própria instituição sob luz natural, com o auxílio de espelho bucal e sonda periodontal, em cadeiras comuns, cadeiras de rodas e camas. Foram também coletados dados demográficos (sexo, idade e grupo étnico) dos idosos. Os dados foram processados e analisados em um programa específico do Projeto SB-2000, Brasil?.

\section{Resultados}

Todas as dez instituições existentes autorizaram a realização do estudo, sendo oito filantrópicas e duas públicas. Dentre os 352 residentes destas instituições, 293 se encontravam na faixa etária de 60 anos ou mais. Destes, dois se recusaram a participar do estudo e outros dois se encontravam hospitalizados, perfazendo 289 o número de idosos examinados. A faixa etária variou de 60 a 103 anos. Aproximadamente a metade (50,5\%) era do sexo masculino e $49,5 \%$ do sexo feminino. Em relação ao grupo étnico, 167 idosos eram brancos, 85 pardos, 28 negros, 8 amarelos e 1 indígena.

A prevalência de cárie foi de $100 \%$, ou seja, todos os idosos apresentaram alguma experiência da doença. Do total de dentes considerados no exame ( $\mathrm{n}=9.248), 519$ $(5,61 \%)$ estavam hígidos, $10(0,11 \%)$ eram apoio de prótese e 8.719 (94,28\%) apresentavam experiência de cárie (dentes $\mathrm{CPO}$ ). O CPOD médio foi 30,17, havendo predomínio do componente perdido, que representou 95,38\% do índice (Tabela 1 ).

Dos 289 idosos, 200 (69,20\%) eram totalmente desdentados e 103 não usavam prótese alguma, representando $35,6 \%$ do total da 
amostra e $51,5 \%$ dos totalmente desdentados. 143 (49,48\%) idosos usavame $232(80,28 \%)$ necessitavam de alguma prótese. A distribuição por arcada e tipo de prótese encontra-se na Tabela 2. Entre aqueles que usavam prótese, a maioria $(45,33 \%)$ usava prótese total na arcada superior e $2457 \%$ na arcada inferior. Em relação à necessidade de prótese, $59,17 \%$ e $51,21 \%$ necessitavam na arcada superior e inferior, respectivamente.
A condição periodontal foi examinada nos idosos dentados, que totalizaram 89 indivíduos (Tabelas 3 e 4). Mais de $60 \%$ dos sextantes foram excluídos por apresentarem menos de dois dentes presentes para a realização do exame. De acordo com o Índice Periodontal Comunitário (CPI), apenas 3 $(3,37 \%)$ dos idosos apresentaram todos os sextantes sadios (Tabela 3). A pior condição mais freqüente foi a presença de cálculo,

Tabela 1 - Índice CPOD e componentes de 289 idosos de instituições de longa permanência em Goiânia-GO, 2003.

Table 1 - DMFT Index and components of 289 elderly individuals living in homes in Goiânia-GO, 2003.

\begin{tabular}{lccccccc}
\hline CPOD e componentes & $\mathrm{n}$ & $\%$ & Média & $\begin{array}{c}\text { Desvio } \\
\text { Padrão }\end{array}$ & Mediana & $\begin{array}{c}\text { Valor } \\
\text { Mínimo }\end{array}$ & $\begin{array}{c}\text { Valor } \\
\text { Máximo }\end{array}$ \\
\hline Cariado & 287 & 3,29 & 0,99 & 2,25 & 0,00 & 0 & 11 \\
Restaurado & 89 & 1,02 & 0,31 & 1,27 & 0,00 & 0 & 9 \\
Restaurado e cariado & 27 & 0,31 & 0,09 & 0,46 & 0,00 & 0 & 4 \\
Perdido por cárie & 8.316 & 95,38 & 28,78 & 6,07 & 32,00 & 3 & 32 \\
Total (CPOD) & 8.719 & 100,0 & 30,17 & 3,87 & 32,00 & 4 & 32 \\
\hline
\end{tabular}

Tabela 2 - Distribuição dos 289 idosos de instituições de longa pemanência em Goiânia-GO, 2003, segundo uso e necessidades de prótese por arcada e tipo.

Table 2 - Distribution of 289 elderly individuals living in homes in Goiânia-GO, 2003 according to use and need of prostheses by arch and type.

\begin{tabular}{|c|c|c|c|c|}
\hline \multirow{3}{*}{ Uso e necessidades de prótese } & \multicolumn{4}{|c|}{ Arcada } \\
\hline & \multicolumn{2}{|c|}{ Superior } & \multicolumn{2}{|c|}{ Inferior } \\
\hline & $\mathrm{n}$ & $\%$ & $\mathrm{n}$ & $\%$ \\
\hline \multicolumn{5}{|l|}{ Uso e tipo de prótese } \\
\hline Não usa & 146 & 50,52 & 201 & 69,55 \\
\hline Usa & 143 & 49,48 & 88 & 30,45 \\
\hline Total & 289 & 100,00 & 289 & 100,00 \\
\hline Prótese parcial removível & 8 & 2,77 & 15 & 5,19 \\
\hline $\begin{array}{l}\text { Uma ou mais próteses fixas e uma ou } \\
\text { mais próteses parciais removíveis }\end{array}$ & 4 & 1,38 & 2 & 0,69 \\
\hline Prótese total & 131 & 45,33 & 71 & 24,57 \\
\hline Total & 143 & 100,00 & 88 & 100,00 \\
\hline \multicolumn{5}{|l|}{ Necessidades e tipo de prótese } \\
\hline Não necessita & 82 & 28,37 & 74 & 25,61 \\
\hline Necessita & 207 & 71,63 & 215 & 74,39 \\
\hline Total & 289 & 100,00 & 289 & 100,00 \\
\hline $\begin{array}{l}\text { Uma prótese fixa ou removível } \\
\text { (um elemento) }\end{array}$ & 23 & 7,96 & 1 & 0,35 \\
\hline $\begin{array}{l}\text { Uma prótese fixa ou removível } \\
\text { (mais de um elemento) }\end{array}$ & 0 & 0 & 48 & 16,61 \\
\hline Combinação de próteses & 13 & 4,50 & 18 & 6,23 \\
\hline Prótese total & 171 & 59,17 & 148 & 51,21 \\
\hline Total & 207 & 100,00 & 215 & 100,00 \\
\hline
\end{tabular}


Tabela 3 - Condição periodontal dos 89 idosos dentados de instituições de longa permanência em Goiânia-GO, 2003, segundo o Índice Periodontal Comunitário (CPI).

Table 3 - Periodontal status of 89 dentate elderly individuals in homes in Goiânia-GO, 2003 according to the Community Periodontal Index (CPI).

\begin{tabular}{lcccc}
\hline $\begin{array}{l}\text { Índice Periodontal } \\
\text { Comunitário (CPI) }\end{array}$ & $\begin{array}{r}\text { Indivíduos, segundo o maior grau de } \\
\text { condição periodontal } \\
\text { observado } \\
\mathrm{n}\end{array}$ & $\%$ & $\mathrm{n}$ & $\%$ \\
\hline Sadio & 3 & 3,37 & 20 & 4,54 \\
Sangramento & 2 & 2,25 & 6 & 1,36 \\
Cálculo & 49 & 55,06 & 128 & 29,02 \\
Bolsa 4-5 mm & 13 & 14,60 & 18 & 4,08 \\
Bolsa 6 mm e + & 2 & 2,25 & 2 & 0,45 \\
Excluído* & 20 & 22,47 & 267 & 60,54 \\
\hline
\end{tabular}

* Menos de 2 dentes presentes $/{ }^{*}$ Less than 2 teeth

Tabela 4 - Condição periodontal dos 89 idosos dentados de instituições de longa permanência em Goiânia-GO, 2003, segundo o Índice de Perda de Inserção Periodontal (PIP). Table 4 - Periodontal status of 89 dentate elderly individuals in homes in Goiânia-GO, 2003 according to the Attachment Loss Index (PIP).

\begin{tabular}{lcccc}
\hline $\begin{array}{l}\text { Perda de Inserção } \\
\text { Periodontal (PIP) }\end{array}$ & $\begin{array}{r}\text { Indivíduos, segundo o maior grau de } \\
\text { condição periodontal observado } \\
\text { n }\end{array}$ & $\%$ & \multicolumn{2}{c}{ Sextantes } \\
\hline $0-3 \mathrm{~mm}$ & 33 & 37,08 & 106 & $\%$ \\
$4-5 \mathrm{~mm}$ & 19 & 21,35 & 43 & 24,04 \\
$6-8 \mathrm{~mm}$ & 16 & 17,98 & 23 & 9,75 \\
$9-11 \mathrm{~mm}$ & 1 & 1,12 & 2 & 0,22 \\
Excluído* & 20 & 22,47 & 267 & 60,54 \\
\hline
\end{tabular}

* Menos de 2 dentes presentes $/{ }^{*}$ Less than 2 teeth

observada em $55,06 \%$ dos indivíduos e $29,02 \%$ dos sextantes. A segunda condição foi a bolsa de 4 a $5 \mathrm{~mm}$, presente em $14,60 \%$ dos indivíduos e $4,08 \%$ dos sextantes. A prevalência de sangramento e bolsa de $6 \mathrm{~mm}$ ou mais foi muito baixa. Quanto ao Índice de Perda de Inserção Periodontal (PIP), predominou a perda de 0 a $3 \mathrm{~mm}$ como pior condição, em $37,08 \%$ dos indivíduos e $24,04 \%$ dos sextantes (Tabela 4). A maior perda observada foi de 9 a $11 \mathrm{~mm}$, em apenas um indivíduo.

Alterações de tecido mole foram encontradas em 39 idosos (13,49\%).

\section{Discussão}

As prevalências de cárie e de edentulismo foram altas nos idosos pesquisados, refletin- do a situação do Brasil como um todo ${ }^{10,11}$. Pinto $^{12}$ afirma que o aumento continuado do índice de cárie com o avançar da idade tem como principal determinante a realização de extrações múltiplas e em larga escala, que se inicia como padrão inevitável a partir dos 30 anos de idade, conforme observado no primeiro levantamento nacional de saúde bucal em $1986^{10}$. De acordo com a revisão realizada por Machado ${ }^{5}$, na maioria dos países em desenvolvimento o índice de cárie e necessidade de prótese em idosos é mais elevado do que nos países desenvolvidos. Nestes, tem havido um aumento no número de idosos que mantêm parte de seus dentes naturais e, conseqüentemente, uma redução significativa no percentual de idosos desdentados.

Comparando-se os dados do presente estudo com os do levantamento nacional de 
$2003^{11}$, observa-se que os índices foram piores. O índice CPO-D foi um pouco superior ao da Região Centro-Oeste e do Brasil como um todo, embora em ambos os estudos o componente perdido tenha representado mais de $90 \%$ do índice. O percentual de idosos que usavam alguma prótese foi mais baixo e o percentual daqueles que necessitavam de alguma prótese foi mais alto do que na Região Centro-Oeste e no Brasil. O predomínio do cálculo dental e das perdas de inserção periodontal de 0 a $3 \mathrm{~mm}$, assim como o alto percentual de sextantes excluídos do exame por escassez de dentes presentes, foram verificados também nos demais estudos ${ }^{11,13-15}$.

A comparação dos dados verificados no presente estudo com os do levantamento nacional ${ }^{11}$ deve ser feita considerando-se que neste último a amostra foi composta por idosos não institucionalizados, examinados em seus domicílios. Como nas instituições de longa permanência públicas e filantrópicas de Goiânia a população atendida é geralmente de nível socioeconômico baixo, é esperada uma situação de saúde mais precária nesta população. Segundo a revisão realizada por Machado ${ }^{5}$, idosos institucionalizados e de baixa renda apresentam maior experiência de cárie, edentulismo, doença periodontal e lesões de tecidos moles do que idosos domiciliados. Além disso, no levantamento nacional foi incluída apenas a faixa etária de 65 a 74 anos.

Comparando-se a condição dentária com a de estudos realizados em idosos institucionalizados de 60 anos ou mais de outros municípios, o quadro é variável. O índice CPOD $(30,17)$ foi comparável ao encontrado em São Paulo $(31,0)^{14}$, São Leopoldo e Porto Alegre $(31,1)^{13}$ e Araraquara $(30,9)^{15}$. A prevalência de edentulismo $(69,2 \%)$ foi mais baixa do que em São Paulo $(84,0 \%)^{14}$ e Araraquara $(72 \%)^{15}$ e mais elevada do que em São Leopoldo e Porto Alegre ${ }^{13}$ (43,1\%) e Barretos $(40 \%)^{16}$.

Alterações de tecido mole foram encontradas em 13,49\% dos examinados. Não se dispõe de dados nacionais e regionais até o momento para comparação, e os estudos locais realizados em instituições não apresentaram informações sobre o número de indivíduos portadores de alterações ${ }^{5}$.

$\mathrm{O}$ alto grau de edentulismo verificado no presente estudo e no Brasil como um todo revela a falta de políticas destinadas à população adulta, que tem caracterizado as ações de saúde bucal. Chama a atenção o alto percentual de idosos totalmente desdentados e que não usavam prótese $(35,6 \%)$, o que pode comprometer a sua qualidade de vida.

Segundo Pinto ${ }^{12}$, com a tendência observada de redução da cárie na dentição permanente em crianças, é esperada uma gradativa mudança de enfoque por parte das ações preventivas e educativas, direcionando-se cada vez mais para outros grupos etários: adolescentes e adultos jovens, infantes ( 0 a 6 anos) e idosos. $O$ estabelecimento da Política Nacional do Idoso ${ }^{8}$ reforça a necessidade de medidas específicas de saúde bucal para este grupo etário, além de ações mais amplas de promoção de saúde que beneficiem toda a população. No caso das instituições de longa permanência, as ações de saúde bucal deveriam ser integradas aos programas de saúde geral, incluindo a reabilitação das condições existentes e buscando proporcionar melhorias na qualidade de vida dosidosos.

\section{Conclusão}

A situação de saúde bucal dos idosos pesquisados é precária, especialmente devido à alta prevalência de cárie e edentulismo. Medidas de promoção e recuperação da saúde são necessárias nas instituições públicas e filantrópicas de longa permanência em Goiânia.

\section{Agradecimentos}

Agradecemos às cirurgiãs-dentistas: Carla Regina S. Marques, Sandra Cristina S. Barbosa, Sandra de Cássia O. Barbosa, Vânia de Fátima D. Batista e suas anotadoras pelo cuidado e apuro técnico nos procedimentos de coleta de dados. 


\section{Referências}

1. Brasil. Lei $\mathrm{n}^{\circ} 10.741$, de $1^{\circ}$ de Outubro de 2003. Estatuto do Idoso. Brasília: Câmara dos Deputados; 2003.

2. Chaimovicz F, Greco DB. Dinâmica da institucionalização de idosos em Belo Horizonte, Brasil. Rev Saúde Pública 1999; 33: 454-60.

3. Born T, Boechat N. A qualidade dos cuidados ao idoso institucionalizado. In: Freitas, EV. Tratado de Geriatria e Gerontologia. Rio de Janeiro: Guanabara Koogan; 2002. p. $768-77$.

4. Colussi CF, Freitas SFT. Aspectos epidemiológicos da saúde bucal do idoso no Brasil. Cad Saúde Pública 2002; 18: 1313-20

5. Machado FR. Saúde bucal do idoso: aspectos epidemiológicos [monografia de especialização]. Goiânia: Faculdade de Enfermagem da Universidade Federal de Goiás; 2001.

6. Mello ALSF. Cuidado odontológico provido a pessoas idosas residentes em instituições geriátricas de pequeno porte em Porto Alegre-RS: a retórica, a prática e os resultados [dissertação de mestrado]. Porto Alegre: Universidade Federal do Rio Grande do Sul; 2001 .

7. Brasil. Ministério da Saúde. Projeto SB-2000 Condições de Saúde Bucal da População Brasileira. Brasília: Ministério da Saúde; 2002.

8. Brasil. Ministério da Justiça. Política Nacional do Idoso. Brasília: Ministério da Justiça, Secretaria Nacional dos Direitos Humanos; 1998.

9. FDI (Fédération Dentaire Internationale). Oral needs of the elderly. Amsterdam: FDI; 1987. Commission on Oral Health. FDI Research and Epidemiology Working Group 5.
10. Brasil. Ministério da Saúde. Levantamento epidemiológico em saúde bucal: Brasil, zona urbana, 1986. Brasília: Ministério da Saúde, Secretaria Nacional de Programas Especiais de Saúde, Divisão Nacional de Saúde Bucal, Fundação Serviços de Saúde Pública; 1988.

11. Brasil. Ministério da Saúde. Projeto SB Brasil 2003 Condições de Saúde Bucal da População Brasileira 2002-2003. Resultados principais. http:// www.cfo.org.br (acessado em 20/Set/2004).

12. Pinto VG. Epidemiologia das doenças bucais no Brasil. In: Kriger, L et al. Promoção de saúde bucal. $3^{\mathrm{a}}$ ed. São Paulo: Aboprev/Artes Médicas, 2003. p. 25-41.

13. Martins Neto M, Padilha DMP. Avaliação clínica da saúde bucal e do uso do fumo de três grupos de idosos. Rev Odont Ciênc 2000; 31: 53-70.

14. Rosa AGF, Fernandez RAC, Pinto VG, Ramos LR. Condições de saúde bucal em pessoas de 60 anos ou mais no Município de São Paulo (Brasil). Rev Saúde Pública 1992; 26: 155-60.

15. Silva SRC, Valsecki Júnior A. Avaliação das condições de saúde bucal dos idosos em um município brasileiro. Rev Panam Salud Publica 2000; 8: 268-71.

16. Zuza EP, Rodrigues RV, Volpiani RC, Toledo BEC. Avaliação das condições bucais de idosos institucionalizados. ROBRAC 2002; 32: 10-12.

recebido em:14/10/04 versão final reapresentada em:12/02/05 aprovado em:15/02/02 